\title{
Cross-over between different symmetries
}

\author{
S. FRAUENDORF* \\ Department of Physics, University Notre Dame, IN 46557, USA \\ *E-mail: sfrauend@nd.edu
}

\begin{abstract}
The yrast states of even even vibrational and transitional nuclei are interpreted as a rotating condensate of interacting d-bosons. The corresponding semi-classical tidal wave concept is used for microscopic calculations of energies and E2 transition probabilities. The strong octupole correlations in the light rare earth and actinide nuclides are interpreted as rotation-induced condensation of interacting f-bosons.

Keywords: d-bosons, f-bosons, cranking model, transitional nuclei, octupole correlations
\end{abstract}

\section{Cross-over between symmetries}

In a macroscopic system the transition between distinct symmetries induced by the change of an external control parameter appears as a phase transition. Fig. 1 schematically illustrates the quantum phase transition between the superconducting (gauge symmetry broken) and normal phases of a type I superconductor. In a small mesoscopic system, as the nucleus, the phase transition is not sharp, instead there is a cross-over region. Starting from the symmetry conserving side, vibrations dynamically violate the symmetry, which become increasingly soft and anharmonic. Starting from the other side, symmetry restoring phenomena, as rotation and tunneling, increasingly lift the degeneracies induced be the broken symmetry. In the case of nuclei, the control parameters are proton and neutron numbers $Z$, $N$, and the angular momentum $I$. The symmetries are the ones of the nuclear mean field. The symmetries of the rotating mean field and the ensuing degeneracies have been discussed in Ref. ${ }^{1}$ In this talk, I will discuss the cross-over between conserved and broken rotational symmetry, as an example for a continuos symmetry, and the cross-over between conserved and broken reflection symmetry, as an example for a discrete symmetry. 

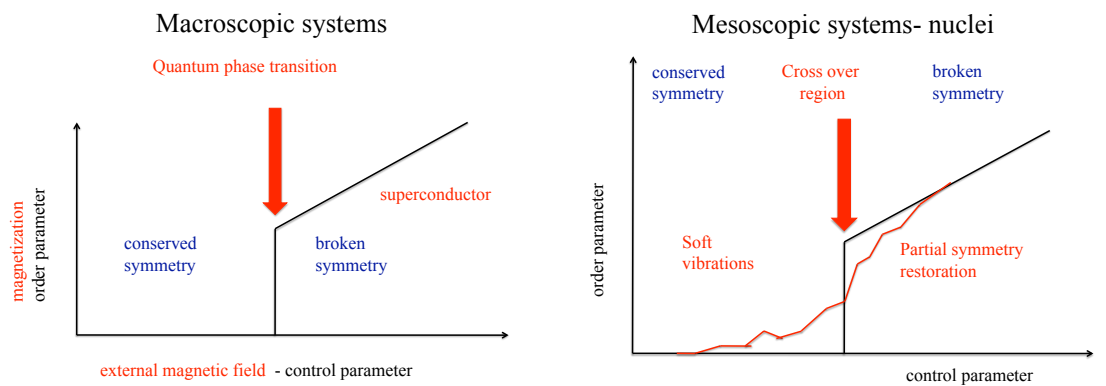

Fig. 1. Quantum phase transition in a Fig. 2. Appearance of an extended crossmacroscopic system. Example: Type I su- over region in a mesoscopic system perconductor at zero temperature.

\section{Condensation of aligned quadrupole phonons-tidal waves}

If the nuclear mean field is spherical, the yrast line is generated by subsequent excitations of particles and holes, where angular momentum is increased by alignment of their spins. The spacing between the yrast levels is irregular, reflecting the single particle energies. If the mean field is deformed, its energy does not depend on its orientation in space, because the Hamiltonian is rotational invariant. This spontaneously broken symmetry is restored by the appearance of rotational states, which represent superpositions of the symmetry broken (oriented) states. The stronger the orientation (order parameter), which is measured by the E2 transition moment, the more rotational $(E=I(I+1) / 2 \mathcal{J})$ the yrast line, and the larger the moment of inertia $\mathcal{J}$. Fig. 3 demonstrates that there is a gradual development from an irregular yrast sequence for the semi-magic $N=82$ Nd-isotope to the rotational sequence of the open shell $N=94$ isotope. In the center of the crossing-over around $N=86$ the yrast energies increase approximately linearly over an extended range of $I$, which is the expected soft vibrational sequence. The yrast line is generated by stacking quadrupole phonons, which align their spins (d-bosons). In other words, there is a condensation of d-bosons. Semi-classically, such a condensate of aligned quadrupole phonons represents a quadrupole wave that travels with the angular velocity $\omega=\Omega_{2} / 2$ over the spherical nuclear surface, where $\Omega_{2}$ is the frequency of the quadrupole vibration. The name "tidal wave" has been suggested ${ }^{2}$ because of its similarity with tidal waves on the ocean.

The surface of a tidal wave moves with the constant angular velocity $\omega$ as the one of a rotor. However there is a difference. The energy and the 

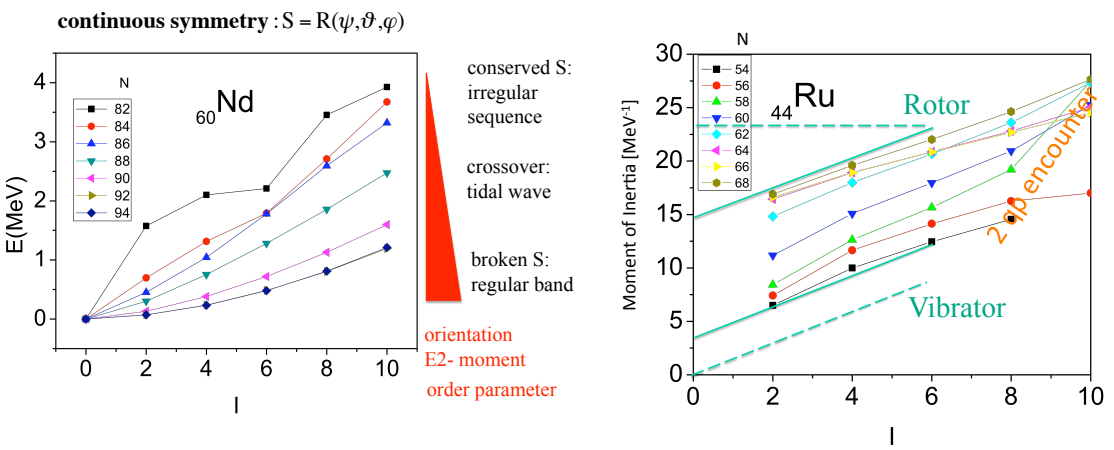

Fig. 3. Yrast energies of the Nd-isotopes. Fig. 4. Moments of inertia of the yrast The red wedge illustrates the deviation from states of the $\mathrm{Ru}$-isotopes spherical symmetry.

angular momentum increase with the amplitude of the harmonic tidal wave wave, whereas the frequency stays constant. The energy and the angular momentum of the rigid rotor increase with the angular frequency while the shape remains unchanged. The difference is manifest by the moment of inertia $\mathcal{J}(I)=I / \omega$, which is proportional to $I$ for the tidal wave and constant for the rotor. Fig. 4 shows the experimental function $\mathcal{J}(I)=$ $2 I /(E(I)-E(I-2))$ the Ru-isotopes. The experiment lies between the limiting cases of a harmonic vibrator (tidal wave) and a rigid rotor. As expected, there is a gradual transition from the vibrational behavior for $N=54$ (center of the cross-over) to rotational one for $N=64$ (broken symmetry).

The interpretation of the near-equidistant yrast sequences as d-boson condensation implies that the reduced transition probability $B(E 2, I \rightarrow$ $I-2$ ) should approximately linearly increase with $I$. The recent lifetime measurements by A. D. Ayangeakaa et al.,$^{3}$ have confirmed the expected increase in ${ }^{102} \mathrm{Pd}$ up to the seven-boson yrast state case. The results are displayed in Fig. 5. The $B(E 2)$ values increase with $I$ in the same way as the moment of inertia, such that their ratio $B(E 2) / \mathcal{J}$ is independent of $I$ within the experimental uncertainties. This clearly demonstrates that the yrast line is generated by stacking d-bosons or, using the semi-classical interpretation, by increasing the amplitude of the tidal wave.

Fig. 5 shows that the yrast line of ${ }^{102} \mathrm{Pd}$ classifies as an anharmonic tidal wave. The moment of inertia is a nearly linear function of $I$ indicated by the line IB (interacting bosons). It deviates from the harmonic limit FB (free bosons) by the small offset at $I=0$, which is a measure of the 

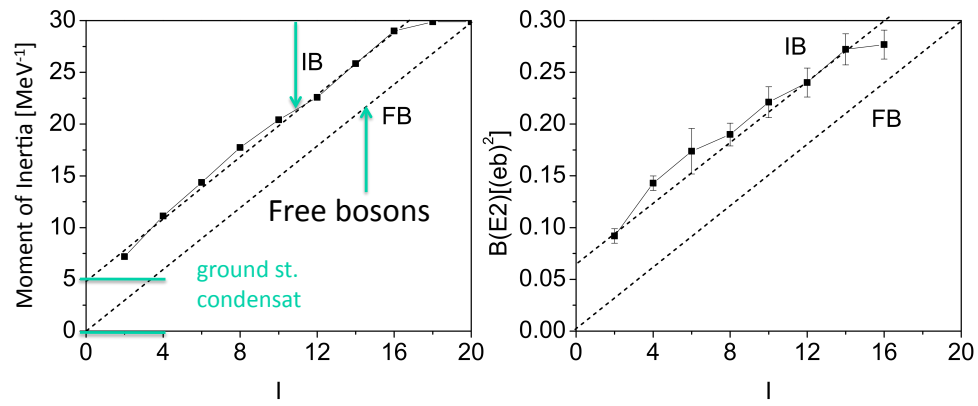

Fig. 5. The moment of inertia $J$ (left) and the $B(E 2, I \rightarrow I-2)$ transition probabilities (right) of the yrast states of ${ }^{102} \mathrm{Pd}$. The dashed line $\mathrm{FB}$ (free bosons) shows the limit of harmonic bosons. The dashed line IB (interacting bosons) illustrates the near linear trend of the interacting bosons.

anharmonicity. The $B(E 2)$ values behave in the same way, such that the ratio $B(E 2) / \mathcal{J}$ is constant. The Liquid Drop Model ${ }^{4}$ suggests that both $B E(2)$ and $\mathcal{J}$ are $\propto \beta^{2}$, which implies that their ratio is $I$-independent. The offset at $I=0$ indicates that the ground state must have some deformation due to fluctuations that are larger than the zero point fluctuations of the harmonic vibrator. In classical terms, the tidal wave starts with a small deformation, which increases along the yrast line. In quantum language, the condensate of interacting bosons rotates like a condensate of free aligned d-bosons to which a small fraction of non-aligned d-bosons is added.

The tidal wave has a static deformed shape in the co-rotating frame of reference. This has lead to the microscopic description suggested by Frauendorf, $\mathrm{Gu}$, and Sun, ${ }^{2}$ which is based on the rotating mean field. We used the SCTAC (shell correction tilted axis cranking) version ${ }^{5}$ of the Cranking Model, which calculates the energy for a given expectation value of the angular momentum operator equal to $I$ by means of the micro-macro method using a deformed Woods-Saxon potential. The energy is minimized with respect to the deformation parameters $\beta$ and $\gamma \cdot{ }^{a}$ Fig. 6 shows that in the case of ${ }^{102} \mathrm{Pd}$, the function $\mathcal{J}(I)$ is very well reproduced. The calculated $B(E 2)$ values show the characteristic increase with $I$. They fluctuate stronger than the experimental values, which is most likely due to the neglected zero point fluctuations of the shape. Note that there are no free parameters adjusted to the experiment. We applied the same method to the even-even nuclides with $44 \leq Z \leq 48$ and $56 \leq N \leq 66 .^{2}$ Deformed solutions were found for

aThere are certain technical problems finding the cranking solution in the nearvibrational regime, which are discussed in Ref. $^{2}$ 

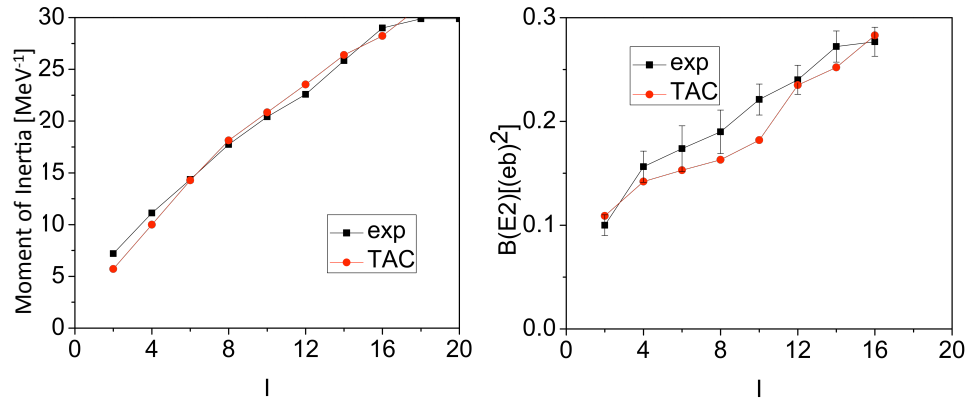

Fig. 6. The moment of inertia $\mathcal{J}$ (left) and the $B(E 2, I \rightarrow I-2)$ transition probabilities (right) of the yrast states of ${ }^{102} \mathrm{Pd}$ compared with the microscopic calculations, which are obtained by minimizing the SCTAC energy (standard LDM, WS potential, and paring) with respect to the deformation parameters $\beta$ and $\gamma$.

$I \geq 2$ even when the solution was spherical for $I=0$. These calculations describe the collective yrast states rather well. They also describe the intrusion of the aligned $\mathrm{h}_{11 / 2}$ two quasi neutron states into the yrast line, which causes the back bending phenomenon seen in most of the studied nuclei.

\section{Condensation of aligned octupole phonons}

So far I have discussed quadrupole deformation, which is reflection symmetric, and the case that the angular momentum is perpendicular to one of the reflection planes. This symmetry implies that the collective sequences (vibrational or rotational bands) have a definite parity and $I$ increases in steps of 2 , i. e. $I=\alpha+2 n$, where $\alpha$ is the signature quantum number of the intrinsic state. If the deformed nuclear shape breaks reflection symmetry such that it still contains two reflection planes and the angular momentum is perpendicular to one of them, then the bands are composed of states with alternating parity, the sequence of which is determined by the simplex quantum number $s$ of the intrinsic state, such that $\pi=s \exp (-i \pi I) .{ }^{4}$ Fig. 7 compares the sequence expected for $s=1$ with the experimental yrast sequences of both parities ${ }^{220} \mathrm{Rn}$. For $I=10-15$ one observes the expected interleaving of states of opposite parity. However, the $\pi=-$ sequence is higher than the $\pi=+$ sequence for $I<10$, and the $\pi=+$ sequence is higher for $I>15$. The Figure also shows the two yrast sequences of a reflection symmetric nucleus that is soft with respect to octupole deformation. In this case the $\pi=-$ yrast sequence is generated by exciting an octupole phonon that aligns its spin of 3 with the total angular momentum (f-boson). Obviously, the low-spin part of the experimental spectrum 


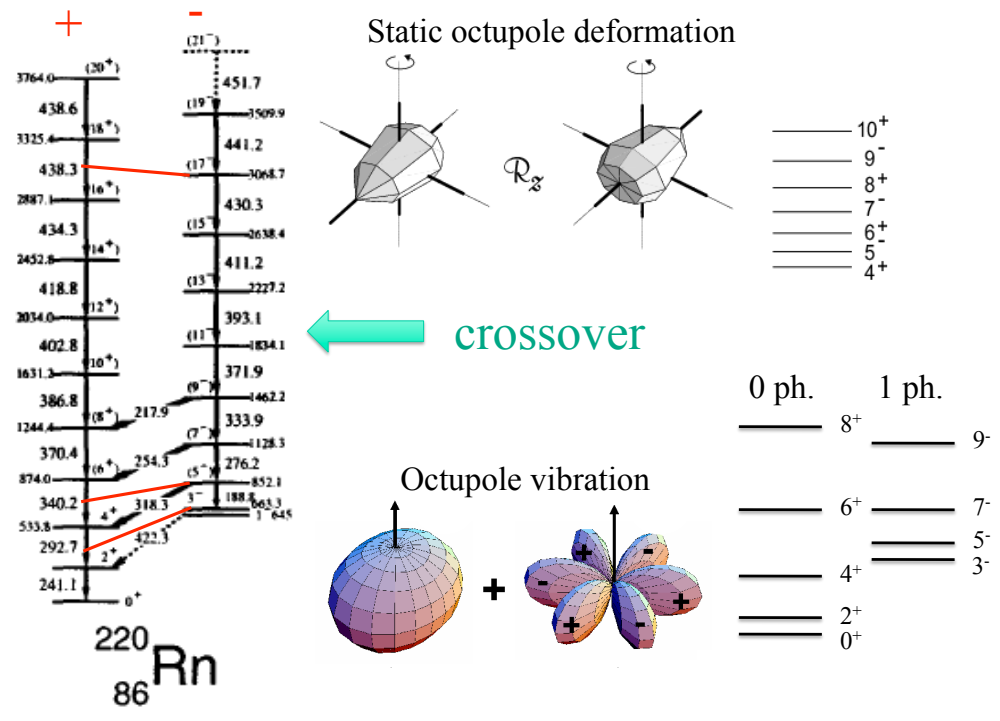

Fig. 7. Octupole vibrations, static octupole deformation as compared to experiment

looks more like the zero and one-phonon bands than the alternating parity sequence of a static octupole shape. In the remainder, I will suggest that the known "octupole deformed" nuclei are situated in the cross-over region between reflection symmetry and asymmetry, where most of them behave like soft anharmonic vibrators.

Fig. 8 illustrates the behavior in cross-over region, which I have discussed in Ref. ${ }^{6}$ In the left panel it is assumed that the octupole vibrations are harmonic and decoupled from the reflection symmetric rotor. The octupole phonons carry $3 \hbar$ of angular momentum, which aligns with the rotational axis. Above the critical frequency $\omega_{c}$ it becomes energetically favorable to excite a phonon instead of further increasing the angular velocity of the rotor, which decreases because the phonon adds $3 \hbar$ of angular momentum. This process is repeated, resulting in a rotation-induced condensation of octupole phonons. Since the octupole phonons have negative parity, the $\pi=+$ and $\pi=-$ yrast sequences change order with their subsequent excitations, which is a hallmark of the condensation. The multi phonon states decay by $E 1$ transitions. The dipole transition operator arises from coupling the isovector $E 3$ moment of the phonons with the isocalar $E 2$ moment of the rotor. As a consequence, only the transitions $(n, I) \rightarrow(n-1, I-1)$ are allowed. The transitions $(n, I) \rightarrow(n+1, I-1)$ are forbidden, because they 

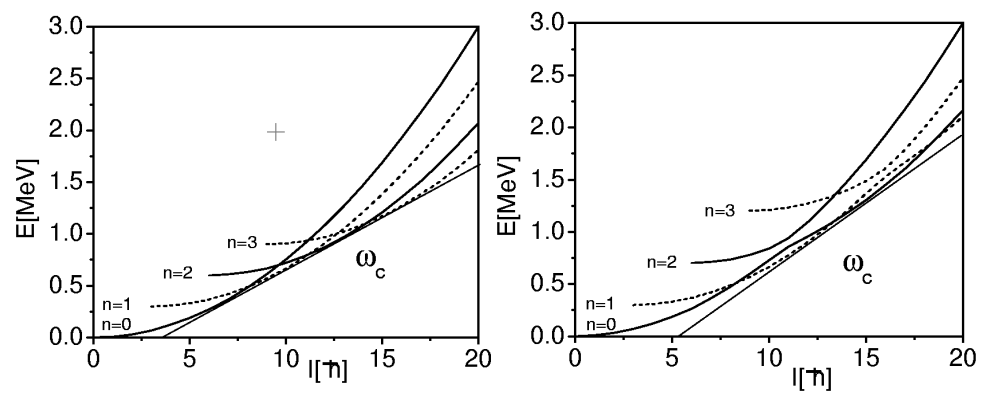

Fig. 8. Energies of aligned octupole phonon bands. Left: harmonic phonons. Right: anharmonic, interacting phonons. The phonon number is $n$.

correspond to a change of rotor angular momentum of $4 \hbar$, which cannot be facilitated by the $E 2$ moment.

The phonons strongly interact in the cross-over region, which causes a repulsion between crossing bands of the same parity. As shown in the right part of Fig. 8, the sharp crossings of the one-phonon with the zeroand two-phonon bands remain, because they have opposite parity. However, the zero- and two-phonon bands mix and repel each other at the avoided crossing. The same holds for the one- and three-phonon bands. As a consequence, the energy difference $S=E_{-}-E_{+}$between between the yrast sequences of both parities oscillates as function of $I$. The experimental energy difference $S(I)=E_{-}(I)-\left(E_{+}(I-1)+E_{+}(I+1)\right)$ for ${ }^{220}$ Ra in Fig. 9 a) display this characteristic pattern. The one-phonon band crosses the zerophonon band before it feels much of the two-phonon band. At the crossing, $S$ changes sign. When the zero-phonon band encounters the tow-phonon one, the two states mix and exchange character (avoided crossing). The level repulsion attenuates the growth of $-S$, which starts decreasing when the $\pi=+$ band has become predominantly the two-phonon state. When the two-phonon band crosses the one-phonon band, $S$ changes sign again. Its growth is attenuated and reversed when the avoided crossing between the one- and three-phonon bands is encountered, the beginning of which is still visible. The angular momentum functions $J_{ \pm}(\omega)$ in Fig. 9 b) reflect the condensation as follows. The $\pi=-$ one-phonon band starts with additional $3 \hbar$ relative to the $\pi=+$ zero-phonon band at the same $\omega$, which is the expected angular momentum carried by an aligned octupole phonon. The difference decreases, when the $\pi=+$ two-phonon band, which carries additional $6 \hbar$, starts mixing into the zero-phonon band. The $\pi=-$ and $\pi=+$ bands have equal angular momentum at the frequency of maximal 
8
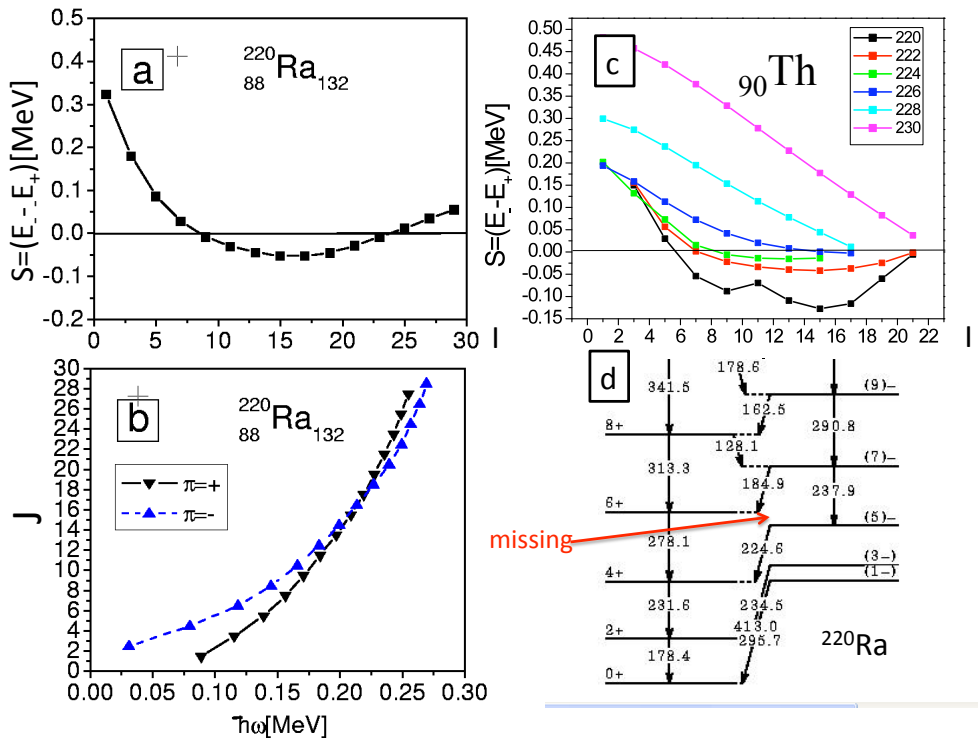

Fig. 9. a, b, c: Experimental energy difference $S=E_{-}-E_{+}$and angular momentum $J(\omega)$ of the $\pi=-$ and $\pi=+$ yrast sequences in ${ }^{220} \mathrm{Ra}$ and the Th isotopes. d: Experimental decay scheme of ${ }^{220} \mathrm{Ra}$.

mixing. Near the one-two-phonon band crossing at $I=24$, where the mixing is small, the angular momentum difference is $-3 \hbar$. Fig. $9 \mathrm{~d}$ ) shows that the forbidden $E 1$ transition $+\rightarrow-$ is indeed missing at low $I$, where the one- and zero-phonon bands are still rather pure. At larger $I$, the transitions $+\rightarrow-$ appear because the two-phonon, from which the transition to the one-phonon band is allowed, starts mixing in.

The aligned octupole phonon is a wave that runs with the angular velocity $\omega_{3}=\Omega_{o c t} / 3$ over the nuclear surface. The rotor rotates with the angular velocity of $\omega_{2}$. If $\omega_{2}=\omega_{3}$ the two motions combine to the rotation of a reflection asymmetric shape (c.f. Fig. 7). The difference $\omega_{-}-\omega_{+}$between the slopes of the two yrast sequences reflects the difference between the angular velocities of the octuople wave and the rotor. The interaction between the octupole phonons, which causes the repulsion between the crossing bands, makes the $\pi= \pm$ yrast sequences more parallel. It tends to lock the two types of motion to the rotation of a stable reflection asymmetric shape, which is non-axial (heart-shaped) in contrast to the conventionally discussed axial octupole deformation (pear-shaped). ${ }^{7}$ Fig. 9 c) shows $S(I)$ for the light $\mathrm{Th}$ isotopes. The $N=134$ and 136 isotopes come closest to the case of static octupole deformation. Mean field calculations predict the 

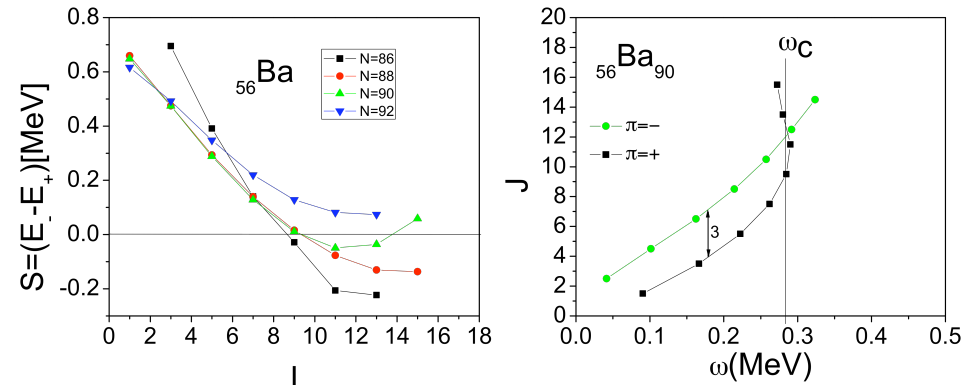

Fig. 10. Experimental energy difference $S=E_{-}-E_{+}$and angular momentum $J(\omega)$ of the $\pi=-$ and $\pi=+$ yrast sequences in the Ba isotopes.

most stable octupole deformation for these neutron numbers. ${ }^{7}$

The other light actinides behave similar to the Th isotopes. Fig. 10 shows the $Z=54 \mathrm{Ba}$ isotopes as an example from the lighter mass region of strong octupole correlations (c.f. ${ }^{7}$ ). The cross-over pattern is clearly seen. As far as studied well enough, the $Z=54,58,60$, and 62 isotones behave in a similar way. For $Z \geq 64$ or $N \geq 94$ the collectivity of the octupole phonons gets lost. The discussed nuclides with strong octupole correlations are not good rotors. They are situated in region of the cross-over toward stable quadrupole deformation, discussed in the preceding section. My discussion assumed a rotor for simplicity. It applies to the tidal waves as well, provided they are anharmonic, such that there is an increase of the angular velocity with $I$. Then the multi-octupole phonon bands are generated by stacking aligned quadrupole phonons onto aligned octupole phonons. The rotationinduced condensation of octupole phonons has been also observed in ${ }^{240} \mathrm{Pu}$, which is a good rotor. ${ }^{8}$

Alternatively, one may interpret the yrast region in terms of a reflectionasymmetric tidal wave that travels over the nuclear surface. This explains why our calculations in the framework of reflection symmetric tidal waves did not well well describe the $Z=58$ - 62, $N=84$ - 90 region. The restoration of parity by tunneling between the shape and its mirror image will cause the splitting between the two yrast sequences of opposite parity. However, the repeated interchange of their order is not obvious from this standpoint. In order to describe the phenomenon in a microscopic way, one should carry out parity-projected cranking calculations of a non-axial, reflection-asymmetric mean field, which is rather challenging. In collaboration with F. Dönau, we have started developing a microscopic approach from the vibrational side. The octupole phonons are described by means 
of the Tamm-Dankoff method in the rotating frame of reference (in oder to avoid the problems of the RPA near the instability). The interaction between the phonons is calculated and a diagonalization with in a space of few phonon excitations will be carried out.

\section{Summary}

The yrast states of transitional nuclei are interpreted in terms of a rotating d-boson condensate, which corresponds a tidal wave running over the nuclear surface. The seven-phonon yrast state has been identified in ${ }^{102} \mathrm{Pd}$. The boson interaction generates anharmonicity, which shows up as a constant shift of the $B(E 2, I \rightarrow I-2)$ value and the moment of inertia $J(I)$ as functions of the spin. The semi-classical tidal wave concept allowed us carrying out microscopic calculations based on the Cranking Model, which reproduce the energies the $B(E 2)$ values without adjustable parameters. All nuclei showing strong octupole correlations are in the cross-over region between reflection symmetry and asymmetry. The properties of their yrast states are more adequately described by the excitation of rotational aligned octupole phonons, which strongly interact, than by a reflection-asymmetric rotor.

\section{Acknowledgments}

Supported by the DoE Grant DE-FG02-95ER4093. I thank A. D. Ayangeakaa and U. Garg for making their lifetime data available to me.

\section{References}

1. S. Frauendorf, Rev. Mod. Phys. 73, 596 (2001)

2. S. Frauendorf, Y. Gu, and J. Sun, Int. J. Mod. Phys. E 20, 425 (2010), online arXiv:1109.1842.

3. D. Ayangeakaa, et al. submitted Phys. Rev. Lett.,

4. A. Bohr and B. R. Mottelson, in Nuclear Structure, Vol. II (Benjamin, New York, 1975).

5. S. Frauendorf, Nucl. Phys. A 677, 115 (2000).

6. S. Frauendorf, Phys. Rev. C 77, 021304 (2007)

7. P. A. Butler, W. Nazarewicz, Rev. Mod. Phys., 68, 349 (1996)

8. X. Wang et al. Phys. Rev. Lett. 102, 122501 (2009) 